\title{
*PROBLEMS FOR RESEARCH WORK IN LEPROSY INSTITUTIONS
}

\author{
T. F. Davey
}

Leprosy is one of the oldest diseases known to mankind, yet until recent years, apart from the discovery of the bacillus by Hansen, it remained one of the most neglected. Now at last the attention of research workers has been drawn to the disease and the last decade has witnessed a tremendous and ever increasing mass of research work designed to elucidate its many problems. So numerous are these that leprosy may almost be considered a research worker's paradise. Whether we refer to its etiology, pathology, symptoms, diagnosis, prognosis, treatment or prevention, every one of these aspects of the disease presents its challenging question marks. It remains almost the only infectious disease of known etiology, the bacillus responsible for which, has never been cultivated with any certainty, though there are those who maintain that the familiar B. leprae is not the causative agent at all.

An exhaustive study of the problems awaiting research in connection with leprosy would occupy a very considerable time and be quite outside the scope of this discussion which is concerned with leprosy work in institutions. I propose therefore to deal briefly with some of the problems which interest me and which I think are of importance to leprologists in Nigeria.

The opportunities for research work in leprosy institutions are of necessity limited. Most of us rather regretfully wish, that after the mind-absorbing routine of every day we could sit down and devote time to some of the practical problems associated with the disease. Some branches of study are totally within the province of the full time expert who is above the reach of clamant patients. We must leave to him such subjects as the cultivation of the bacillus, animal inoculation with human leprosy and the academic but very interesting study of the relation of leprosy tn the ductless glands, especially with regard to the pigmentary changes associated with the disease. It is very unlikely that there is opportunity for studies such as these in Leprosy Institutions in Nigeria. What subjects are then available?

1. The Study of Cases of Leprosy

In the first place I think that there is an urgent need for the

* Paper read at the Leprosy Conference, Enugu, S.E. Nigeria, August 28th-3oth, I939. 
simple straighforward study of cases of the disease, not on one occasion only, but on repeated occasions. Although found throughout the world, leprosy shows not inconsiderable changes in its manifestations from one country to another, and up to the present, throughout the literature, comparative studies have one serious lack, namely that Nigeria is excluded. All of us have a lot still to learn simply from the study of cases, and this needs to be pursued both in the laboratory and in the field. Such a study must be associated with what is the most important practical problem in connection with leprosy, namely the recognition of infectious cases.

\section{(a) Laboratory Investigations.}

All will agree that the most important practical problem associated with leprosy is, "which are infectious cases?" It is only when we know which cases are responsible for the spread of the disease that we can embark with confidence on any scheme of control. This question is as yet unsettled. It was included in the preliminary agenda for discussion at the Cairo Conference, but received scant attention there. The generally accepted view is that all cases that are bacteriologically positive on routine examination of the skin and nasal mucosa, may be considered as infectious. This very simple and easily proved criterion is in practice not as simple as it seems. Let us consider the different types of leprosy.

Commencing with neural leprosy, all are familiar with the resistant individuals in whom the disease takes the form of tuberculoid macules, commonly accompanied with nerve enlargement. These people are uniformly bacteriologically negative except when in a state of reaction, when they may be harbouring numerous bacilli in the lesions. This is a temporary phase and often initiates the process of resolution. Are these cases to be regarded as infectious at this time? If so, we have to agree to the existence of temporary infectious phases in leprosy. This is not a very important matter in the case of tuberculoid cases, as reacting tuberculoids are not very common, but it is of great importance in connection with other manifestations of the disease.

The next group of leprosy lesions comes under the heading of pale, flat macules, and together with the tuberculoid lesions completes, for practical purposes, the picture of neural leprosy in Nigeria. I have reason to believe that cases which exhibit neuritis without the appearance of macules are rare in this country, and are not of practical importance.

Pale flat macules constitute a most heterogeneous group of 
lesions, the largest single group in Nigeria. On the one hand they include all tuberculoid lesions which are resolving for these elevated lesions must pass through a flat stage in the process of resolution. Leaving these on one side there remain all those lesions which are flat, hypopigmented, and exhibiting signs of nerve involvement. You may see an individual who presents a few large macules, discrete, clearly outlined, with a hypopigmented edge, well marked sensory loss in the lesions, and often return of the pigment and sensation in the centre of the macules. These cases, though lacking the massive cell response of the tuberculoids, are resistant and almost invariably bacteriologically negative. Their course is very chronic, and there is a marked tendency to self-healing. On the one hand, one sees not infrequently the person who is literally covered with small flat macules which spread radially, show little or no healing, and ultimately coalesce with neighbouring macules. Finallv almost the entire body surface may be covered, and it is possible, if the sensory changes are not realised, for them to be considered not cases of leprosy at all, but simply individuals with a pale skin. These cases are not invariably bacteriologically negative, especially in the nose. The former type may revert to this type if the resistance of the individual deteriorates.

Turning to the lepromatous manifestations of the disease, no difficulty arises with nodular and diffuse forms. The resistance of these people is negligible and the skin teems with bacilli. One and all may be considered infectious. It is worthy of note that, as cases are considered in which the process is less active and the thickening of the skin less pronounced, a point is reached when the thickening is very slight. Hypopigmentation is not invariable in these cases, and it is easily possible for such a case to be overlooked altogether.

When we turn to cases with lepromatous macules, the problem of infectiousness rises to its full intensity, and in this connection the Cairo Conference is not very helpful. Its definition of the leproma includes the following: " as a rule lepromatous lesions are more ill-defined and diffusely outlined than the leprides, and they do not exhibit the same tendency to radial extension or the same changes of colour and sensation." This definition overlooks many cases of leprosy in Nigeria. It is an everyday experience to come across individuals who give a history of the sudden onset of numerous hypopigmented macules. At their onset these are usually very small and numerous, clearly hypopigmented, sometimes erythematous but almost invariably possessing a characteristic ruddy colour, due in all probability to minor degrees of erythema. 
The lesions present a multitude of appearances, sometimes raised, sometimes flat. I hey are commonly, but not necessarily associated with thickenning of the ears, and the skin is usually thickened. To the casual observer they may appear identical in appearance with pale flat macules or even with tuberculoid plaque lesions, but they are invariably positive bacteriologically. Anaesthesia is very common, but is ill-defined, not limited to the lesions. The skin between the lesions is also usually bacteriologically positive. As the disease progresses, the thickening of the skin may decrease. At the same time coalescence of the lesions occurs, and at last a point is reached where they become indistinguishable from the coalescing type of pale flat macule, and exhibit widespread pallor, frequent nerve involvement, and a slightly positive bacteriological result, often only on repeated examination.

Now lepromatous lesions have a characteristic histology, endothelial proliferation around blood capillaries in the neighbourhood of the infecting focus of bacilli being the first step. These endothelial cells together with local connective tissue elements become macrophages which are unable to cope with the rapid proliferation of the bacilli, and the typical lepra cells are produced. These cells, some vacuolated, others containing masses of bacilli maybe in a state of symbiosis, constitute a characteristic picture. This appearance is uniform in nodular and cliffuse cases, but what of lepromatous macules?

Turning to neural lesions, Wade and others maintain that these also have a uniform histology of an essentially tuberculoid nature, marked in major tuberculoid cases, slight in pale flat macules. This appearance is distinct from the leproma.

We have seen that clinically and bacteriologically the two great sub-divisions of leprosy meet, and somewhere at their junction lies the dividing mark of the infectious from the uninfectious case. If the two types have a distinct histology, what is the histology of those cases near to the concurrence of the two types? These are the cases which need further elucidation. There is a great need for a clinical, histological and bacteriological study of these cases which are between the lepromatous, acknowledged infectious cases on the one hand and the neural, uninfectious cases on the other. If only infectious cases are to be segregated, it is essential to know on which side of the line these cases stand. The Lepromin Test may be of considerable assistance in the elucidation of this problem. There is no record of its use in Nigeria, and an unexplored field of research is awaiting investigation in connection with it.

We have, however, not yet exhausted the possible lines of research concerning the infectiousness of leprosy. Granted the 
existence of borderline cases, is it not at least possible that there occur temporary infectious phases in cases which may be bacteriologically negative on a single examination? In S.E. Nigeria I have been impressed with the fall in the general resistance of the public to infectious diseases which occurs at certain times in the year, particularly between the months of May and July. The diet is poor, consisting largely of cassava, and the onset of the wet season brings mosquitoes, and with them malaria as a further factor in decreasing resistance. Oberdorffer has suggested that a periodic variation in resistance occurs, and this inevitably has a bearing on leprosy. The resistance of the individual is the key to the problem, and it is reasonable to expect that a lowered resistance may induce a case which is just above the threshold of infectiousness to cross that threshold and become infectious for a time, returning over the threshold when his resistance is improved.

It would be of interest to test the Sedimentation Index of a large group of people at intervals through the year and so ascertain the extent and time of the lowered resistance which is believed to occur.

\section{(b) Field Investigations.}

The other great sphere for the study of cases of leprosy is the village where the leper lives. A multitude of possible lines of research is available here. Survey work is in the nature of research and gives a complete picture of leprosy in an area such as the study of cases in an institution never can give.

Statistics relating to incidence, childhood rates, sex rates, type rates, contact rates can be assessed, and there are as yet scarcely any adequate data of this sort relating to leprosy in Nigeria. The observation of untreated cases over a period, the following up of contacts, the establishing of sources of infection, all these matters are of importance and can only be carried out in and around the homes of lepers.

Two other subjects remain for investigation in field studies.

(a) Reference has already been made to the bacteriologically positive case who presents little or no obvious thickening of the skin and also no hypopigmentation. Their existence among the general public would vitiate any scheme of control unless they were discovered. It would be of considerable interest to take a limited area in a neighbourhood where leprosy is known to be rife and test everybody bacteriologically. It is only by such a study that these cases could be revealed.

(b) In India, children suffer from a form of leprosy which is of great interest and which mainly consists of vague pale macules 
which are often of a temporary nature only. Vague pale macules from numerous causes are found in Nigeria, but the possibility of leprosy being responsible in some cases needs to be investigated.

\section{The Study of the Factors comprising the Resistance of THE INDIV IDUAL TO LEProsy}

Returning to the Institution, the next great consideration awaiting study is that of the resistance of the patient to infection with leprosy. There can be no doubt that the key which opens the door to recovery in every case is the building up of resistance, and that is our primary aim in treatment. Many factors are concerned, for example, hygiene, exercise, intercurrent disease, and diet. The relation of leprosy to craw-craw, to worm infections, to avitaminosis, to malaria, to anaemia, all these things need working out. It may be that we should emphasise one factor more than another in treatment. Certainly an institution gives one an admirable opportunity for the study of leprosy in relation to diet. This is an important matter in Southern Nigeria, where the diet of the people often leaves much to be desired. This and similar studies are of considerable importance because the significance of the resistance of the individual in the progress of the disease cannot be exaggerated.

\section{The Treatment of Leprosy}

Quite "apart from the building up of the resistance of the patient so that he offers a barren soil to the bacillus, innumerable methods of specific treatment are possible. None of those available at present can be regarded as entirely satisfactory, and there is room for a great deal more research in this direction. Such research was especially advocated by the Cairo Conference, particularly with the use of dyes such as fluorescin. Leprosy Institutions offer the best field for studies of this description, which should be directed not only towards the discovery of a specific remedy for the disease, but also the relief of its complications, trophic ulcers, etc.

There are various other highly interesting fields for research in regard to leprosy, the problem of Lepra Reaction for instance still remaining in doubt, but the lines of study which have been indicated are, I consider, those which should first exercise the minds of leprologists in Nigeria. 